\title{
Targetry Issues for the Fermilab 2-MW Neutrino Superbeam
}

\author{
M.A. Kostin, V.I. Balbekov, N.V. Mokhov, Z. Tang
}

FNAL, Batavia,IL 60510, USA

\begin{abstract}
The possibility to use various target materials are studied for a $2 \mathrm{MW}$ neutrino superbeam facility that can be built at Fermilab utilizing the Main Injector and a Proton Driver. A simple target solution found is a thick graphite rod taking a broad proton beam.
\end{abstract}

\section{MOTIVATION}

The neutrino oscillation is a hot topic nowadays. The oscillation is only possible when the neutrino is a massive particle. The Standard Model does not allow the neutrino to have mass, therefore observation of the neutrino oscillation opens window into the physics beyond the Standard Model. The resent results show that the neutrinos do oscillate. The SuperKamiokande experiment has provided a very strong evidence that the muon neutrino experience large flavor changing transition [1]. As shown $v_{\mu}$ are transformed primarily to $v_{\tau}$. The SNO has reported that the solar electron neutrinos are changing their flavor to $v_{\mu}$ and/or $v_{\tau}$ [2] and the Large Mixing Angle solution is preferable.

Despite the fact that many of oscillation parameters will be determined more precisely by forthcoming experiments such as MINOS, MiniBooNE and others, some questions still will be out of reach of those. For example, the problems of neutrino mass hierarchy, $\mathrm{CP}$ violation in the leptonic sector and probably CPT violation can only be addressed by an experiment of the next generation, socalled neutrino superbeam facility. Such an experiment is expected to utilize a conventional neutrino beam of a very high intensity and a detector with fiducial mass of $20 \mathrm{kton}$ and more. Whereas the detector and beamline issues are relatively well known, the target remains the most critical one. For instance, the FNAL Main Injector coupled with a Proton Driver would provide a proton beam of $2 \mathrm{MW}$ on target. A target solution for such a facility is not obvious.

\section{CRITERIA FOR A GOOD TARGET}

A good target for a neutrino superbeam facility would satisfy the following conditions: it survives one spill; the steady state temperature is relatively low; the target life time is greater than 6 months; in the case of multiple choice for a target candidate, the optimal target is the one that provides the highest $\pi^{+}$yield in the energy interval of interest. The first three clauses are quite obvious. The last one is driven by the physics case. The main goal of such a facility would be a measurement of the $v_{\mu} \rightarrow v_{e}$ oscillation parameters. For a $v_{\mu}$-beam coming from a NuMI-like facility, the $v_{e}$ appearance probability to leading order is given by

$P_{v_{\mu} \rightarrow v_{e}}=\sin ^{2}\left(\theta_{23}\right) \sin ^{2}\left(2 \theta_{13}\right) \sin ^{2}\left(\frac{1.27 \Delta m_{32}^{2}\left(e V^{2}\right) L(k m)}{E_{v}(G e V)}\right)$

A detector must be placed at an appropriate distance from neutrino source to let the neutrino enough time to oscillate. For example, one of possible locations for the detector site is the Homestake mine at $1290 \mathrm{~km}$ from Fermilab.

The main source of $v_{\mu}$ is the two body pion decay $\pi^{+} \rightarrow v_{\mu} \mu^{+}$. The pions are produced in interactions of the initial proton beam with a target. Neutrino energy from the above decay is

$$
E_{v}=\frac{0.43 E_{\pi}}{1+\gamma^{2} \theta^{2}}
$$

where $\gamma$ is the pion relativistic boost and $\theta$ is the neutrino emission angle at decay. It is seen from (2) that in order to get a neutrino beam of the energy of $2.5-3.5 \mathrm{GeV}$ in a detector placed on the facility axis, one would need to focus pions of 5.8 to $8.2 \mathrm{GeV}$ energy. For an off-axis detector, the energy interval of pions should be extended up to $20 \mathrm{GeV}$ [3].

\section{MATERIAL CHOICE}

\section{What survives one spill ?}

A number of commonly used materials was considered for a target. The target was simulated with the MARS 14 code [4]. We assumed that a $120 \mathrm{GeV}$ proton beam hits a rod target with a length of two interaction lengths. For the first trial, the beam was assumed to be a Gaussian with $\sigma_{x}=\sigma_{y}=1 \mathrm{~mm}$ (approximately NuMI parameters). The target transverse size was optimized for the maximal pion yield scanning the radius of target with a step of $0.5 \mathrm{~mm}$ starting from $2.5 \mathrm{~mm}$ (see Table 1). In order to achieve a power of $2 \mathrm{MW}$, the nominal NuMI 
TABLE 1. Density of peak energy deposition and pion yield in the $5.95-8.05 \mathrm{GeV}$ interval for various targets. Also shown are the optimal target radii $R_{T}$ for $\sigma=1 \mathrm{~mm}$.

\begin{tabular}{c|l|l|l}
\hline Material & $\begin{array}{l}\text { Peak ED } \\
\text { density }(\mathrm{J} / \mathrm{g})\end{array}$ & $\begin{array}{l}\pi^{+} \text {yield } \\
(\mathrm{N} / \mathrm{POT})\end{array}$ & $\begin{array}{l}\text { optimal } \\
\mathrm{R}_{T}(\mathrm{~mm})\end{array}$ \\
\hline Graphite & $1581 \pm 18$ & $0.326 \pm 0.005$ & 5.0 \\
\hline Nickel & $6520 \pm 251$ & $0.311 \pm 0.004$ & 2.5 \\
\hline Inconel & $6011 \pm 259$ & $0.311 \pm 0.004$ & 3.0 \\
\hline Copper & $6084 \pm 216$ & $0.310 \pm 0.004$ & 3.0 \\
\hline Indium & $5248 \pm 149$ & $0.336 \pm 0.004$ & 3.0 \\
\hline Mercury & $10064 \pm 293$ & $0.324 \pm 0.004$ & 3.0 \\
\hline
\end{tabular}

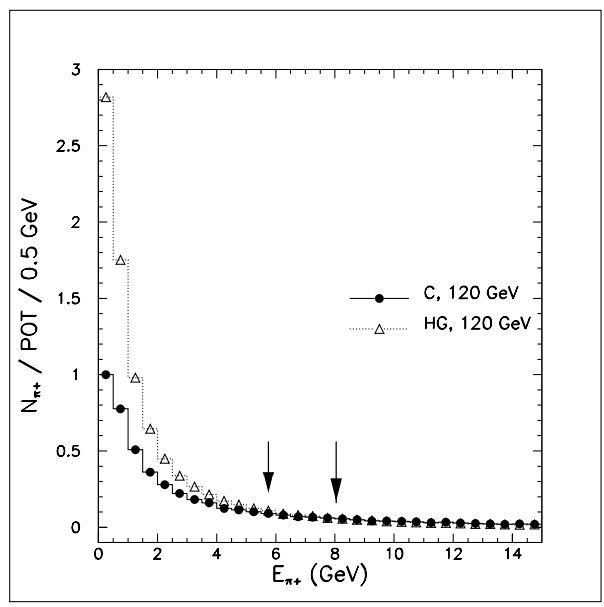

FIGURE 1. Pion yield versus pion energy. The arrows restrict the interval of our interest.

beam intensity is rescaled by a factor of 5 that corresponds to $2 \times 10^{14}$ protons per spill. We also assumed that the Main Injector repetition period of $1.9 \mathrm{~s}$ does not change.

Calculated peak energy deposition densities on a beam axis are shown in Table 1 for $\mathrm{C}$ through $\mathrm{Hg}$ targets. For the beam conditions described above all the materials experience a significant thermal shock. The stress limits known for graphite, nickel and inconel are about $1000 \mathrm{~J} / \mathrm{g}$. The limit for copper is about $600 \mathrm{~J} / \mathrm{g}$. As one can see from the table, none of the solid materials can survive such conditions.

Despite the fact that the above conditions are not operable for the solid materials, it is interesting to notice that the pion yield in the defined energy interval does not vary significantly with the target material (Table 1). Fig. 1 shows the pion yield versus pion energy for graphite and mercury. Much more soft pions are produced in a mercury target. But what is important, the yield is about the same in the region of interest for on-axis beams, $5.95 \leq E_{\pi} \leq 8.05 \mathrm{GeV}$. Pion spectra for the other materials studied behave similarly.

The most obvious idea of stress reduction is to increase

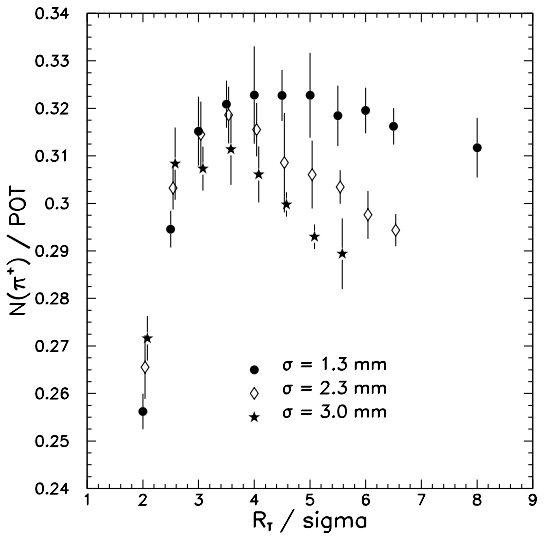

FIGURE 2. Number of $\pi^{+}$coming from a graphite target in the interval $5.95-8.05 \mathrm{GeV}$ against the value of target radius over beam sigma. Shown are dependencies for different beam RMSs. The distributions are normalized per the number of protons on target (POT).

the beam transverse spot size. In the study below, both the beam spot size $\sigma=\sigma_{x}=\sigma_{y}$ and target radius $R_{T}$ are varied.

For a graphite target the pion yield as a function of $R_{T} / \sigma$ is shown in Fig. 2 for different $\sigma$. As one can see the maximal yield does not change much with $\sigma$. This is due to the fact that pions leave the target at much larger angle than a specific angle at which the target collimates the pions, that is $\operatorname{atan}\left(\mathrm{R}_{T} / \lambda_{I}\right) \approx 1^{\circ}$ (Fig. 3). Energy deposition in the hottest cell of target is acceptable at $\sigma>1.5 \mathrm{~mm}$ (Fig. 4). For example, for $\sigma=3 \mathrm{~mm}$ and target radius $R_{T}$ of $9 \mathrm{~mm}$ the peak energy deposition is $310 \mathrm{~J} / \mathrm{g}$ that is below the limit. Thus the beam spot size and target radius for graphite can be substantially increased compared to the NuMI parameters without significant loss of the yield.

The same idea does not work well for solid dense materials. An attempt to bring the energy deposition safely below the limit by increasing $\sigma$ and $R_{T}$ results in a substantial reduction of the pion yield. For example, if one increases the beam $\sigma$ up to $12.5 \mathrm{~mm}$ keeping the ratio to be optimal $R_{T} / \sigma=2.5$, the pion yield reduces down to 0.22 pions per proton that is much smaller than the yield for an optimal graphite target of about 0.33 . The density of peak energy deposition is $1250 \mathrm{~J} / \mathrm{g}$ for inconel in this case that is still above the limit.

The studies performed show that graphite is the most convenient material for a FNAL neutrino superbeam facility. Indeed, the dense solid materials do not provide enough of pion flux at the acceptable beam and target radii. The use of light materials with large interaction lengths such as $\mathrm{Li}$ and $\mathrm{Na}$ will lead to a too long target, making focusing of secondaries problematic. A mercury jet target seems to be too complex, expensive and haz- 


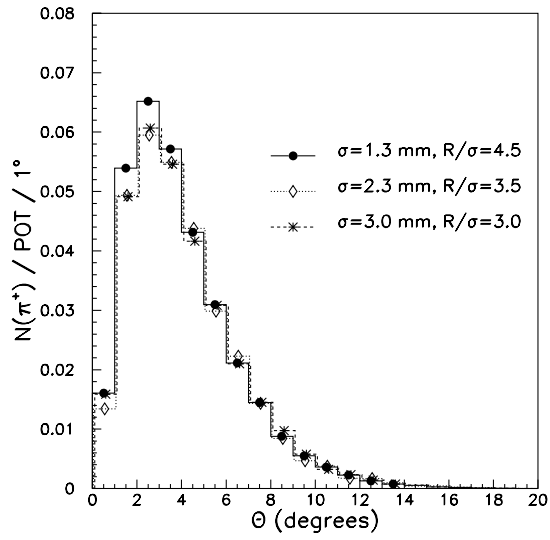

FIGURE 3. Angular distributions for pions coming off a graphite target. $\theta$ is an angle between the target axis and pion direction.

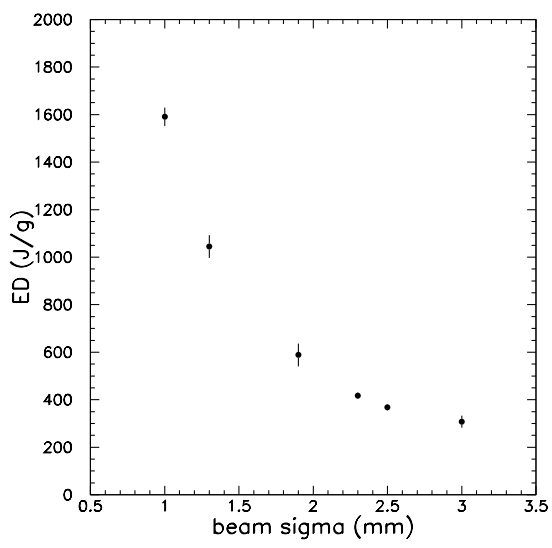

FIGURE 4. Energy deposition in the hottest cell of a graphite target versus beam spot size.

ardous device and at the same time it does not provide any advantages over a graphite target for the given experimental conditions.

\section{Target life-time}

One of the factors limiting the target life-time is radiation damage. The life-time determined here corresponds to the time when $5 \mathrm{dpa}$ (displacements per atom) occur in the hottest cell of the target. The atoms are displaced due to interactions with hadrons with kinetic energy of $>0.1 \mathrm{MeV}$. The $5 \mathrm{dpa}$ limit corresponds to the integrated hadron flux of $5 \times 10^{22} \mathrm{~cm}^{-2}$. From the MARS simulations we have found that the limit of $5 \mathrm{dpa}$ is reached within 5 years and 8 months assuming 10 months of operation per year at the full intensity.

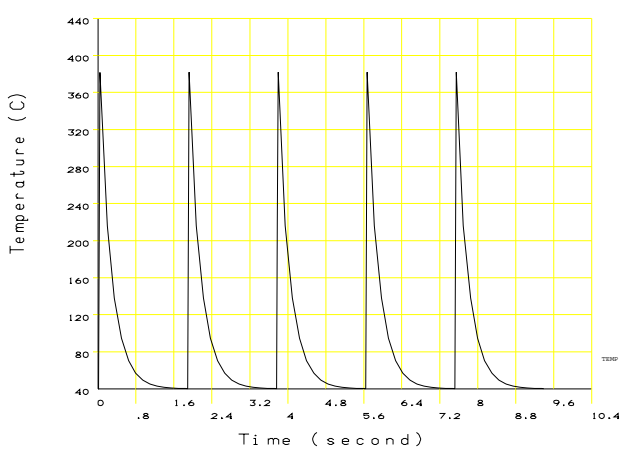

FIGURE 5. Temperature evolution in the hottest cell of a graphite target with $1.9 \mathrm{~s}$ repetition rate.

\section{Temperature buildup}

The temperature evolution has been investigated with the ANSYS code [5]. The temperature on the side target surface was fixed to $40^{\circ} \mathrm{C}$. As Fig. 5 shows, the temperature in the hottest cell oscillates between $40^{\circ} \mathrm{C}$ and $380^{\circ} \mathrm{C}$ without any buildup. The tensile stress obtained from the simulation is about $20 \mathrm{MPa}$ at the tensile strength for graphite of about $90 \mathrm{MPa}$.

\section{CONCLUSIONS}

A graphite target represents a simple and cheap solution for the FNAL neutrino superbeam facility. Such a target satisfies all the requirements applied: it survives one spill; radiation damage does not constrain the life-time significantly; there is no temperature buildup in target; pion yield is quite high.

\section{REFERENCES}

1. Y. Fukuda et al., Evidence for oscillation of atmospheric neutrinos. Phys. Rev. Lett. 81 (1998) 1562.

2. Q. R. Ahmad et al., Direct evidence for neutrino flavor transformation from neutral current interactions in the Sudbury Neutrino Observatory. e-Print Archive: nucl-ex/0204008.

3. A. Para and M. Szleper, Neutrino oscillations experiments using Off-axis NuMI beam. e-Print Archive: hepex/0110032.

4. N. V. Mokhov, "The MARS Monte Carlo", Fermilab FN-628 (1995); N. V. Mokhov and O. E. Krivosheev, "MARS Code Status", Fermilab-Conf-00/181 (2000); http://www-ap.fnal.gov/MARS/.

5. "ANSYS (rev.5.1)”,Swanson Analysis System, Inc., SASI/DN-P511:51, Houston (1994). 